\title{
Management of Vataja abhishyanda (Allergic conjunctivitis) with Gutika anjana and Punarnavadi eye drops: A comparative clinical study
}

\author{
Research Article
}

\section{Shashi Prakash Gupta1 ${ }^{*}$, Vaghela D B ${ }^{2}$}

1. PhD Scholar, 2. Associate Professor

Department of Shalakya Tantra, I.T.R.A, GAU, Jamnagar, Gujarat, India.

\begin{abstract}
Background: Vataja Abhishyanda is characterized by Toda (Pricking pain), Sangharsha (foreign body sensation), Achchasruta (watery discharge), Alpa Shopha (mild chemosis), Vishushka Bhava (feeling of dryness), Parushya (roughness) etc which are very similar to the most of signs and symptoms of the Allergic Conjunctivitis. It is one of the most common type of eye allergy and is widely experienced by global population. Aims and Objective: . To evaluate and compare the efficacy of Punarnavadi eye drops and Gutika Anjana in the management of Vataja Abhishyanda(Allergic conjunctivitis). Materials and methods: Total 104 patients diagnosed with symptoms and signs of Vataja Abhishyanda were selected from the outpatient department of Shalakya tantra. The selected patients were assigned randomly into two groups, group A (Punarnavadi eye drop) having 51 and group B (Gutika Anjana) having 50 patients. Duration of treatment was 8weeks with follow up for one month after the trial. Result: In Group A, $96.08 \%$ got complete relief after the completion of treatment, $03.92 \%$ got marked improvement. In Group B, $98 \%$ got complete relief after the completion of treatment, 2\% got marked improvement. Conclusion: Gutika Anjana shows better relief in all sign and symptoms on the basis of percentage. The reason may be Anjana has maximum absorption due to more contact of time with the tissue which is responsible for better bioavailability.
\end{abstract}

Key Words: Allergic conjunctivitis; Gutika Anjana; Punarnavadi eye drop; Vataja Abhishyanda.

\section{Introduction}

Vataja Abhishyanda is characterized by Toda (Pricking pain), Sangharsha (foreign body sensation), Achchasruta (watery discharge), Alpa Shopha (mild chemosis), Vishushka Bhava (feeling of dryness), Parushya (roughness) etc which are very similar to the most of signs and symptoms of the Allergic Conjunctivitis. So, Vataja Abhishyanda can be corelated with Allergic Conjunctivitis. Allergic conjunctivitis is the most common type of eye allergy and is widely experienced by global population. It has an equal distribution more or less throughout the world, without any exception to the developed and under developed countries(1). The epidemiology of ocular allergic diseases has not been sufficiently investigated to date. In general, ocular allergy is estimated to affect $5-22 \%$ of the population, depending on the geographical setting and on the age of the population(2).

General lines of treatment explained by our Acharya for Abhishyanda are: Langhana, Tikta Anna Sewana, Alepan, Swedana, Siravedhana, Virechana, Anjana and Aschyotana(3). Among these Anjana and

* Corresponding Author:

Shashi Prakash Gupta

PhD Scholar,

Department of Shalakya Tantra,

I.T.R.A, GAU, Jamnagar,

Gujarat. India.

Email Id: shashiprakash.5887@gmail.com
Ashchyotana are simple, safer, most inexpensive procedure, which are indicated in eye diseases.

In Sushruta Samhita Uttaratantra Vataabhishyanda Pratishedha(Su.Utt.9/15), Gutika Anjana is indicated for Vataja Abhishyanda. It contains Gairika, Saindhava, Pippali and Shunthi. All the drugs have Madhura Vipaka and Snigdha Guna and most of the drugs are Vatashamaka in properties. So, this formulation is selected for current topic.

Punarnavadi Eye Drops(Anubhoota Yoga) containing drugs Punarnava Moola, Mishri, Sphatika and Saindhava has known for its efficacy to manage eye conditions like redness, pain, watering, foreign body sensation etc. which are the complaints of the disease Abhishyanda. The name Punarnavadi Eye Drops is given as the Punarnava is contributing as main drug.

\section{Aim of the study}

To evaluate and compare the efficacy of Punarnavadi eye drops and Gutika Anjana in the management of Vataja Abhishyanda(Allergic conjunctivitis).

\section{Materials and Methods}

The patients attending the O.P.D. and I.P.D. of Dept.of Shalakyatantra, I.P.G.T. \& R.A., G.A.U., Jamnagar and referred from other O.P.D were selected irrespective of their sex, religion, caste, occupation etc. Informed and written consent were taken from all the patients. 
Shashi Prakash Gupta et.al., A comparative clinical study on gutika anjana and Punarnavadi eye drops in vataja abhishyanda

The study was open labelled randomized comparative clinical trial. The patients were selected by Computerized simple random sampling method.

The diagnosis of Vataja Abhishyanda (Allergic Conjunctivitis) was done on the basis of clinical picture as per the Modern and Ayurvedic texts. For this purpose a special research proforma was prepared as per the modern and Ayurvedic view. After taking ophthalmic and systemic history, a detailed conjunctival examination was carried out by slit lamp examination before and after study. The study was approved by Institutional ethics committee letter no. PGT/7 A/Ethics/ 2017-18/3042 dated 19/02/18. The Clinical trial was registered under CTRI with CTRI No. CTRI/ 2018/02/012159 registered on 26/02/2018.

\section{Statistical analysis}

On the basis of grading scores the effect of therapy in subjective parameters was assessed with the help of Wilcox an signed rank test and objective parameters were assessed with paired ' $t$ ' test. The comparison was done between the groups in subjective parameters with Mann Whitney test and objective parameters with unpaired ' $\mathrm{t}$ ' test. The results obtained were considered highly significant for $P<0.001$, significant for $P<0.05$ and insignificant for $P>0.05$.

\section{Inclusion criteria}

1. Patients willing for the treatment.

2. Patients between 18 to 50 years of age irrespective of sex, caste and religion.

3. Patients presenting with signs and symptoms like Toda ( Pricking pain), Stambha (Stiffness of lids), Sangharsha (Foreign body sensation), Parushya (Roughness), Shiroabhitapa(Headache), Vishushka Bhava (Dryness - feeling), Shishirasruta (Cold lacrimation), Achchasruta (clean/ watery discharge), Alpa Shopha (Mild chemosis) and on examination hyperaemia, swelling, follicle and papillae in palpebral and hyperaemia and chemosis in bulbar conjunctiva was diagnosed as Vataja Abhishyanda (Allergic Conjunctivitis).

\section{Exclusion criteria}

1. Patients below 18 years and above 50 years

2. Patients having complications of corneal involvement like marginal corneal ulcer, dacryocystitis, trachoma, keratoconjunctivitis etc., other types of allergic conjunctivitis like giant papillary conjunctivitis, etc., Infective conjunctivitis, non-specific systemic infections, patients of ocular trauma, uncontrolled diabetes mellitus, patients having genetic predisposition to allergy.

\section{Study design}

The selected patients were assigned randomly into two groups (A \&B), group A having 51 and group $\mathrm{B}$ having 50 patients. Each group was subjected to the treatment in the following method. [Table 3]
Table 1: Contents of Punarnavadi eye drops (Anubhoota Yoga)

\begin{tabular}{|c|c|c|c|c|}
\hline No. & Drug Drug & $\begin{array}{c}\text { Botanical } \\
\text { Name Name }\end{array}$ & $\begin{array}{c}\text { Part } \\
\text { used } \\
\text { used }\end{array}$ & $\begin{array}{c}\text { Propor } \\
\text { tion }\end{array}$ \\
\hline 1 & Punarnava & $\begin{array}{c}\text { Boerhavia } \\
\text { diffusa } \text { Linn. }\end{array}$ & Root & 1 Part \\
\hline 2 & Mishri & $\begin{array}{c}\text { Sugar(English } \\
\text { name) }\end{array}$ & - & $1 / 2$ Part \\
\hline 3 & Sphatika & $\begin{array}{c}\text { Alum(English } \\
\text { name) }\end{array}$ & - & $1 / 2$ Part \\
\hline 4 & Saindhava & $\begin{array}{c}\text { Sodii } \\
\text { chloridum }\end{array}$ & - & $1 / 2$ Part \\
\hline
\end{tabular}

Table 2: Contents of Gutika Anjana (Su.Ut.9/15)

\begin{tabular}{|c|c|c|c|c|}
\hline No. & Drug & Botanical Name & $\begin{array}{l}\text { Part } \\
\text { used }\end{array}$ & $\begin{array}{l}\text { Propo } \\
\text { rtion }\end{array}$ \\
\hline 1 & Shunthi & $\begin{array}{r}\text { Zin } \\
\text { officino }\end{array}$ & Rhizome & 8 Part \\
\hline 2 & 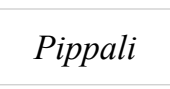 & $\begin{array}{l}\text { Piper longum } \\
\text { Linn. }\end{array}$ & Root & Part \\
\hline 3 & Saindhava & Sodii chlorid & - & 2 Part \\
\hline 4 & Gairika & $\begin{array}{l}\text { Ochre(English } \\
\text { name) }\end{array}$ & - & 1 Part \\
\hline
\end{tabular}

Table 3: Posology

Group A:

Punarnavadi eye drops (Anubhoota Yoga)

Group B: Gutika Anjana

(Su.Ut.9/15)

\begin{tabular}{|c|c|c|}
\hline & (Anubhoota Yoga) & (Su.Ut.9/15) \\
\hline Form & Arka & Anjana with water \\
\hline Dose & $\begin{array}{c}2 \text { drops } 4 \text { times per day } \\
\text { both eyes }\end{array}$ & $\begin{array}{l}\text { 60mg per day in } \\
\text { two divided doses } \\
\text { both eyes }\end{array}$ \\
\hline $\begin{array}{l}\text { Method of } \\
\text { application }\end{array}$ & $\begin{array}{l}\text { Kaninaka Sandhi } \\
\text { (Nasal canthus) }\end{array}$ & $\begin{array}{l}\text { applying from } \\
\text { nasal to temporal } \\
\text { canthus. }\end{array}$ \\
\hline $\begin{array}{l}\text { Contact } \\
\text { period }\end{array}$ & 3-5 minutes & $\begin{array}{l}\text { till watering and } \\
\text { irritation subsides }\end{array}$ \\
\hline $\begin{array}{c}\text { After } \\
\text { application }\end{array}$ & $\begin{array}{l}\text { Mopping by sterile } \\
\text { cotton }\end{array}$ & $\begin{array}{c}\text { Netra } \\
\text { Prakshalana (eye } \\
\text { wash) with } \\
\text { distilled water }\end{array}$ \\
\hline $\begin{array}{c}\text { Route of } \\
\text { Administra } \\
\text { tion }\end{array}$ & Topical (Netra) & Topical (Netra) \\
\hline Duration & 8 weeks & 8 weeks \\
\hline Time & $\begin{array}{l}\text { Morning, afternoon, } \\
\text { evening and at bed } \\
\text { time }\end{array}$ & $\begin{array}{l}\text { Morning and } \\
\text { evening }\end{array}$ \\
\hline
\end{tabular}

Follow up: Follow up study was done after 1 month after completion of trial.

\section{Assessment of therapy}

Grading and scoring system was adopted for assessing the following clinical feature before the commencement of trial and after completion of trial.

\section{Subjective assessment criteria \\ Toda ( Pricking pain)}

Absent - no Toda

Occasionally present and very mild

Intermittently present and mild 
Frequently present and moderate - not

disturbing the routine work

Present almost all the time - severe -

disturbing routine activities

3

\section{Stambha (Stiffness of lids)}

Absent - no Stambha

Occasionally feeling of Stambha of lids. Intermittently and mild feeling of Stambha of lids

Frequently and moderate feeling of Stambha of lids

Feeling of Stambha of lids and Eye ball

almost all the time.

\section{Sangharsha (Foreign body sensation)}

Absent - no Sangharsha

Occasionally present, mild and not

troublesome

Intermittently present mild and troublesome

Frequently present, moderate and

troublesome

Present almost all the time, severe and

continuously troublesome

\section{Parushya (Roughness)}

Absent- no Parushya

Visible dryness of lid skin with no Parushya, mild (fade) dull white streak after scratching on to lid skin, disappearing after sometime. Dryness with Parushya, bright white streak on scratching on to the lid skin remaining for a considerable time

Dryness, Parushya, slight thickening of the lid skin with visible crisscross marking with no cracking of the lid skin. Dryness, Parushya, slight thickening of the lid skin with visible crisscross marking with cracking of the lid skin.

\section{Vishushka Bhava (Dryness - feeling)}

Absent - no Vishushka Bhava

Occasionally present and very mild

Vishushka Bhava

Intermittently present and mild Vishushka

Bhava

Frequently present moderate Vishushka

Bhava

Vishushka Bhava present almost all the time and severe

\section{Shiroabhitapa (Headache)}

Absent- no Shiroabhitapa

Noticeable Shiroabhitapa

Moderate Shiroabhitapa

Severe Shiroabhitapa

\section{Shishirasruta (Cold lacrimation)}

Absent-no Shishirasruta

Mild and occasionally need to wipe

Mild but intermittently need to wipe

Moderate and need to wipe frequently

Severe and need to wipe almost all the time.

\section{Achchasruta (clean/ watery discharge)}

Absent - no Achchasruta

Mild and occasionally need to wipe

Mild but intermittently need to wipe

Moderate and need to wipe frequently

Severe and need to wipe almost all the time.

\section{Alpa Shopha (Mild chemosis)}

Absent - no Alpa Sopha

Puffy lids with discrete mild Alpa Sopha

visible on slit lamp examination

Puffy lids with discrete mild Alpa Sopha

visible on tourch light examination

Swelling of lids visibly evident Alpa Sopha

Swollen eye lids / ballooning of conjunctiva.

Jantunam Iva Sarpanam (Crawling sensation)

Absent - no feeling of Jantunam Iva

Sarpanam

Occasional feeling of Jantunam Iva

Sarpanam

Intermittent feeling of Jantunam Iva

Sarpanam

Frequent feeling of Jantunam Iva Sarpanam

continuous feeling of Jantunam Iva Sarpanam

\section{Nimeshonmeshana Krichchata (Difficulty in lid movement)}

Absent - no Nimeshonmeshana Krichchata 0

Occasional Nimeshonmeshana Krichchata 1

Intermittent and mild Nimeshonmeshana

Krichchata

Frequent and moderate Nimeshonmeshana

Krichchata

Nimeshonmeshana Krichchata almost all the time

\section{Kshiadhamana Iva Bhati (Feeling of distended} eyes)

Absent - no Akshiadhamana Iva Bhati

Occasional Akshiadhamana Iva Bhati

Intermittent Akshiadhamana Iva Bhati

Frequently Akshiadhamana Iva Bhati

Akshiadhamana Iva Bhati almost all the time.

\section{Shushka Dushika (Dry discharge)}

Absent - no Shushka Dushika

Occasional Shushka Dushika need not to wipe,

Intermittent need to wipe Shushka Dushika, 2 Frequent need to wipe Shushka Dushika, All the time need to wipe Shushka Dushika

\section{Alpa Dushika (Scanty discharge)}

\section{Absent - no Alpa Dushika}

Occasional Alpa Dushika need not to wipe, 1 Intermittent need to wipe s Alpa Dushika, 2 Frequent need to wipe Alpa Dushika, All the time need to wipe Alpa Dushika 
Shashi Prakash Gupta et.al., A comparative clinical study on gutika anjana and Punarnavadi eye drops in vataja abhishyanda

Chala Ruja (Referred pain)

Absent - no Chala Ruja

Bhru Ruja or 1 site involved

Bhru, Shankha, Ruja or 2 sites involved

Bhru, Shankha, Lalata, Ruja or 3 sites

involved

Bhru, Shankha, Lalata, Shiro Ruja or 4 sites involved

\section{Kandu (Itching)}

Absent - no Kandu

Occasional tickle sensation not requiring to

rub eye

Intermittent tickle sensation not requiring to

rub eye

Continuous itch which requires rubbing of

eyes

An incapacitating itch which would require

significant eye rubbing

\section{Ragata (Redness)}

Absent - no Ragata

Mild - occasional Ragata with clear pattern

of blood vessels

Moderate - intermittent Ragata with clear

pattern of blood vessels

Moderate - Ragata with the disturbed

patterned of blood vessels

Severe - Velvety conjunctiva with loss of

pattern of blood vessels

\section{Daha(Burning sensation)}

Absent - no Daha

Occasional Daha not requiring to rub eye

Intermittent Daha not requiring to rub eye

Continuous Daha which requires rubbing of

eyes

An incapacitating Daha which would require significant eye rubbing

\section{Objective assessment}

Hyperemia in Palpebral Conjunctiva

No Manifestation

Dilatation of vessels in lower palpebral

conjunctiva

Dilatation of vessels in lower part of upper

palpebral conjunctiva

Dilatation of vessels in lower and upper

palpebral conjunctiva

\section{Swelling in Palpebral Conjunctiva}

No Manifestation

Localized oedema

Diffused mild oedema

Diffused marked oedema

\section{Follicle}

No Manifestation

1-9 follicles

10-19 follicles

20 or more follicles

\section{Papillae}

No Manifestation

Diameter $0.1-0.2 \mathrm{~mm}$

Diameter 0.3- $0.5 \mathrm{~mm}$

Diameter $\geq 0.6 \mathrm{~mm}$

\section{Hyperemia in Bulbar Conjunctiva}

No Manifestation

Dilatation of vessels in any two quadrants of bulbar conjunctiva

Dilatation of vessels in any three quadrants

of bulbar conjunctiva

Dilatation of vessels in all four quadrants of bulbar conjunctiva

\section{Chemosis in Bulbar Conjunctiva}

No Manifestation

Micro chemosis

Macro chemosis

Bullous chemosis of entire conjunctiva

\section{Observations and results}

Demographic data have been presented for 104 patients, while clinical data was made on 101 patients who completed the trial, and similarly, the results were analyzed and are presented in Tables 4 to 12 .

\section{Demographic profile}

Age wise distribution of patients showed that maximum $48.08 \%$ patients came under the age group of $18-30$ years , $32.70 \%$ between $31-40$ years and $19.23 \%$ belonged to $41-50$ years age group. Sex wise distribution showed that $59.61 \%$ were female and rest patients were male i.e. $40.38 \%$. Incidence of Vataja Abhishyanda was found $37.50 \%$ in house wives followed by $24.03 \%$ in serviceman. $21.15 \%$ in workers while $17.30 \%$ in students. Majority of the patients i.e. $52.88 \%$ belonged to lower middle class while $43.26 \%$ were from middle class and $03.84 \%$ were from upper middle class. Maximum number of the patients i.e. $95.19 \%$ had seasonal problem, $80.76 \%$ had sunlight, $70.19 \%$ had wind, $59.61 \%$ had problem in hot season, $44.23 \%$ had pollens, $25.96 \%$ had dust and $11.53 \%$ had cold things as aggravating factor. Maximum patients i.e. $67.30 \%$ had allergic rhinitis and $2.88 \%$ patients had eczema as associated complaint. Maximum i.e. 56.73\% patients were of Vata Pitta Prakriti, followed by 24.04\% patients of Pitta-Kaphaja Prakriti, while $19.23 \%$ were of Vata-Kaphaja Prakriti.

\section{Clinical profile}

The study showed that maximum $98.08 \%$ patients had chief complaint Achchasruta, $86.54 \%$ patients had Shushka Dushika, 84.61\% patients had Sangharsha, Jantunam Iva Sarpanam and Alpa Dushika, 83.65\% patients had Toda, 72.11\% patients had Akshyadhmantam Iva Bhati, $70.19 \%$ patients had Shiroabhitapa, 69.23\% patients had Vishushka Bhava, $56.73 \%$ patients had Stambha, 49.04\% patients had Alpa Shopha, $46.15 \%$ patients had Chala Ruja, 35.58\% patients had Parushya, $23.08 \%$ patients had Shishirasruta and $14.42 \%$ patients had Nimeshonmeshana Krichchata as chief complaint. Maximum $81.73 \%$ patients had Kandu, 38.46\% patients had Ragata and $23.08 \%$ patients had Daha as associated symptoms. 


\section{Effect of therapy in Group A}

In this group significant relief was found in Toda (97.17\%), Stambha (97.47\%), Sangharsha (98.39\%), Parushya (96.08\%), Shiroabhitapa $(96.30 \%)$, Vishushka Bhava (97.09\%), Shishirasruta (97.56\%), Achchasruta (96.77\%), Alpa Shopha (96.97\%), Jantunam Iva Sarpanam (98.36\%), Nimeshonmeshana Krichchata (93.33\%), Akshyadhmantam Iva Bhati
(97.65\%), Shushka Dushika (97.08\%), Alpa Dushika (96.83\%), Chala Ruja (96.00\%), Kandu (95.93\%), Ragata (93.48\%), Daha (96\%).

$91.78 \%$ relief was found in hyperemia, $95.55 \%$ in swelling, $55.55 \%$ in follicle, $70 \%$ in papillae in palpebral conjunctiva and $94.44 \%$ in hyperemia in Bulbar Conjunctiva.

Table 4: Effect of Punarnavadi eye drop (Group A) on chief complaints of 51 patients.

\begin{tabular}{|c|c|c|c|c|c|c|c|c|c|c|}
\hline \multirow{2}{*}{ Chief complaints } & \multicolumn{2}{|c|}{ Mean } & \multirow{2}{*}{ Diff } & \multirow{2}{*}{$\begin{array}{l}\% \text { of } \\
\text { relief }\end{array}$} & \multicolumn{6}{|c|}{ Signed rank test } \\
\hline & BT & AT & & & SD & SE & $\mathbf{n}$ & W & p & Remarks \\
\hline Toda(Pricking pain) & 2.65 & 0.07 & 2.58 & 97.17 & 0.67 & 0.10 & 40 & 820 & $<0.001$ & HS \\
\hline Stambha (Stiffness of lids) & 2.82 & 0.07 & 2.75 & 97.47 & 0.64 & 0.12 & 28 & 406 & $<0.001$ & HS \\
\hline Sangharsha (Foreign body sensation) & 2.69 & 0.04 & 2.65 & 98.39 & 0.56 & 0.08 & 46 & 1081 & $<0.001$ & HS \\
\hline Parushya(Roughness) & 2.55 & 0.10 & 2.45 & 96.08 & 0.51 & 0.11 & 20 & 210 & $<0.001$ & HS \\
\hline Shiroabhitapa(Headache) & 2.38 & 0.08 & 2.30 & 96.30 & 0.57 & 0.09 & 34 & 595 & $<0.001$ & HS \\
\hline Vishushka Bhava(Dryness - feeling) & 2.94 & 0.08 & 2.86 & 97.09 & 0.64 & 0.10 & 35 & 630 & $<0.001$ & HS \\
\hline Shishirasruta(Cold lacrimation) & 2.73 & 0.06 & 2.67 & 97.56 & 0.72 & 0.18 & 15 & 120 & $<0.001$ & HS \\
\hline Achchasruta (clean/watery discharge) & 3.03 & 0.09 & 2.94 & 96.77 & 0.70 & 0.09 & 51 & 1326 & $<0.001$ & HS \\
\hline Alpa Shopha (Mild chemosis) & 2.44 & 0.07 & 2.37 & 96.97 & 0.56 & 0.10 & 27 & 378 & $<0.001$ & HS \\
\hline $\begin{array}{l}\text { Jantunam Iva Sarpanam (Crawling } \\
\text { sensation) }\end{array}$ & 2.65 & 0.04 & 2.61 & 98.36 & 0.53 & 0.07 & 46 & 1081 & $<0.001$ & HS \\
\hline $\begin{array}{l}\text { Nimeshonmeshana Krichchata } \\
\text { (Difficulty in lid movement) }\end{array}$ & 2.57 & 0.14 & 2.43 & 93.33 & 0.53 & 0.20 & 7 & 28 & $<0.05$ & $\mathrm{~S}$ \\
\hline $\begin{array}{l}\text { Akshyadhmantam Iva Bhati (Feeling of } \\
\text { distended eyes) }\end{array}$ & 2.29 & 0.05 & 2.24 & 97.65 & 0.64 & 0.10 & 37 & 703 & $<0.001$ & HS \\
\hline Shushka Dushika (Dry discharge) & 2.97 & 0.08 & 2.89 & 97.08 & 0.64 & 0.09 & 46 & 1081 & $<0.001$ & HS \\
\hline Alpa Dushika (Scanty discharge) & 2.86 & 0.09 & 2.77 & 96.83 & 0.60 & 0.09 & 44 & 990 & $<0.001$ & HS \\
\hline Chala Ruja (Referred pain) & 2.50 & 0.10 & 2.40 & 96.00 & 0.59 & 0.13 & 20 & 210 & $<0.001$ & HS \\
\hline
\end{tabular}

BT- Before treatment, AT- After treatment, SD- Standard deviation, SE- Standard error, n- number of patients, WWilcox an signed rank test, p- Probability, HS- highly significant, S- significant

Table 5: Effect of Punarnavadi eye drop (Group A) on associated symptoms of 51 patients

\begin{tabular}{|c|c|c|c|c|c|c|c|c|c|c|}
\hline \multirow{2}{*}{ Associated symptoms } & \multicolumn{2}{|c|}{ Mean } & \multirow{2}{*}{ Diff } & \multirow{2}{*}{$\begin{array}{l}\% \text { of } \\
\text { relief }\end{array}$} & \multicolumn{6}{|c|}{ Signed rank test } \\
\hline & BT & AT & & & SD & SE & n & $\mathbf{W}$ & $\mathbf{p}$ & Remarks \\
\hline Kandu (Itching) & 2.73 & 0.11 & 2.62 & 95.93 & 0.57 & 0.08 & 45 & 1035 & $<0.001$ & HS \\
\hline Ragata (Redness) & 2.55 & 0.16 & 2.39 & 93.48 & 0.50 & 0.11 & 18 & 171 & $<0.001$ & HS \\
\hline $\begin{array}{c}\text { Daha } \\
\text { (Burning sensation) }\end{array}$ & 2.77 & 0.11 & 2.66 & 96.00 & 0.50 & 0.16 & 9 & 45 & $<0.05$ & $\mathrm{~S}$ \\
\hline
\end{tabular}

Table 6: Effect of Punarnavadi eye drop (Group A) on objective criteria of 51 patients

\begin{tabular}{|c|c|c|c|c|c|c|c|c|c|c|c|}
\hline \multirow{2}{*}{\multicolumn{2}{|c|}{ Objective criteria }} & \multicolumn{2}{|c|}{ Mean } & \multirow{2}{*}{ Diff } & \multirow{2}{*}{$\begin{array}{l}\% \text { of } \\
\text { relief }\end{array}$} & \multicolumn{6}{|c|}{ Paired $t$ test } \\
\hline & & BT & AT & & & SD & SE & $\mathbf{n}$ & $\mathbf{T}$ & p & Remarks \\
\hline \multirow{4}{*}{$\begin{array}{c}\text { Palpebral } \\
\text { Conjunctiva }\end{array}$} & Hyperemia & 2.92 & 0.24 & 2.68 & 91.78 & 0.51 & 0.07 & 50 & 36.96 & $<0.001$ & HS \\
\hline & Swelling & 2.25 & 0.10 & 2.15 & 95.55 & 0.58 & 0.13 & 20 & 16.37 & $<0.001$ & HS \\
\hline & Follicle & 1.00 & 0.44 & 0.56 & 55.55 & 0.52 & 0.17 & 9 & 3.16 & $<0.05$ & $\mathrm{~S}$ \\
\hline & Papill & 1.08 & 0.32 & 0.76 & 70.00 & 0.48 & 0.07 & 46 & 10.75 & $<0.001$ & HS \\
\hline \multirow{2}{*}{$\begin{array}{c}\text { Bulbar } \\
\text { Conjunctiva }\end{array}$} & Hyperemia & 2.84 & 0.15 & 2.69 & 94.44 & 0.57 & 0.09 & 38 & 28.80 & $<0.001$ & HS \\
\hline & Chemosis & 0.00 & 0.00 & 0.00 & 0.00 & 0.00 & 0.00 & 0 & 0.00 & 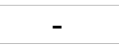 & 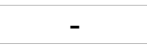 \\
\hline
\end{tabular}

\section{Effect of therapy in Group B}

In this group significant relief was found in Toda (98.15\%), Stambha (98.63\%), Sangharsha (97.92 \%), Parushya (97.37\%), Shiroabhitapa (97.75\%), Vishushka Bhava (98.95\%), Shishirasruta (95.83 \%), Achchasruta (96.30 \%), Alpa Shopha (98.11\%), Jantunam Iva Sarpanam (97.92 \%), Nimeshonmeshana Krichchata (94.11\%), Akshyadhmantam Iva Bhati (98.83 \%), Shushka Dushika (98.30 \%), Alpa Dushika (99.14 \%), Chala Ruja (98.41 \%), Kandu (97.90 \%), Ragata (96\%), Daha (91.66\%).

$95.65 \%$ relief was found in hyperemia, $100 \%$ in swelling, $75 \%$ in follicle, $83.67 \%$ in papillae in palpebral conjunctiva and $94.28 \%$ in hyperemia in Bulbar Conjunctiva. 
Shashi Prakash Gupta et.al., A comparative clinical study on gutika anjana and Punarnavadi eye drops in vataja abhishyanda

Table 7: Effect of Gutika Anjana (Group B) on chief complaints of 50 patient

Chief complaints

Toda(Pricking pain)

Stambha (Stiffness of lids)

Sangharsha (Foreign body

sensation)

Parushya(Roughness)

Shiroabhitapa(Headache)

Vishushka Bhava(Dryness - feeling)

Shishirasruta(Cold lacrimation)

Achchasruta (clean/ watery

discharge)

Alpa Shopha (Mild chemosis)

Jantunam Iva Sarpanam (Crawling

sensation)

Nimeshonmeshana Krichchata

(Difficulty in lid movement)

Akshyadhmantam Iva Bhati (Feeling

of distended eyes)

Shushka Dushika (Dry discharge)

Alpa Dushika (Scanty discharge)

Chala Ruja (Referred pain)

\begin{tabular}{|c|c|}
\hline \multicolumn{2}{|c|}{ Mean } \\
\hline BT & AT \\
\hline 2.40 & 0.04 \\
\hline 2.43 & 0.03 \\
\hline 2.46 & 0.05 \\
\hline 2.37 & 0.06 \\
\hline 2.40 & 0.05 \\
\hline 2.74 & 0.03 \\
\hline 2.66 & 0.11 \\
\hline 2.81 & 0.10 \\
\hline 2.52 & 0.04 \\
\hline 2.46 & 0.05 \\
\hline 2.42 & 0.14 \\
\hline 2.38 & 0.02 \\
\hline .78 & 0.04 \\
\hline .72 & 0.02 \\
\hline 2.42 & 0.03 \\
\hline
\end{tabular}

$\%$ of

\section{relief}

98.15

$2.40 \quad 98.63$

SD SE

Signed rank test

2.4197 .92

0.64

$0.49 \quad 0.09$

$0.54 \quad 0.08$

0.60

\begin{tabular}{l|l|}
2.35 & 97.75 \\
\hline
\end{tabular}

\begin{tabular}{l|l}
2.71 & 98.95
\end{tabular}

\begin{tabular}{l|l}
0.58 & 0.09
\end{tabular}

\begin{tabular}{l|l}
0.71 & 0.12 \\
\hline
\end{tabular}

\begin{tabular}{l|l}
0.71 & 0.12 \\
1.01 & 0.33
\end{tabular}

Signed rank test

\begin{tabular}{l|l}
2.55 & 95.83
\end{tabular}

0.71

0.10

$2.70 \quad 96.30$

0.51

0.11

2.41

98.11

0.54

0.08

\begin{tabular}{l|l}
2.28 & 94.11
\end{tabular}

\begin{tabular}{l|l}
0.48 & 0.18
\end{tabular}

$2.36 \quad 98.83$

0.63

0.10

2.74

98.30

0.66

0.10

2.70

99.14

0.67

0.49

0.10

0.09

\begin{tabular}{|l|l|}
\hline n & 1035
\end{tabular}

30465

$<0.001$

$<0.001$

39780

\begin{tabular}{|l|l|}
16 & 136 \\
\hline
\end{tabular}

\begin{tabular}{l|l}
37 & 703 \\
\hline
\end{tabular}

\begin{tabular}{l|l}
35 & 630
\end{tabular}

$<0.001$

$<0.001$

$<0.001$

$1176<0.001$

$231<0.001$

$780<0.001$

$<0.001$

$<0.05$

Table 8: Effect of Gutika Anjana (Group B) on associated symptoms of 50 patients

\begin{tabular}{|c|c|c|c|c|c|c|c|c|c|c|}
\hline \multirow{2}{*}{ Associated symptoms } & \multicolumn{2}{|c|}{ Mean } & \multirow{2}{*}{ Diff } & \multirow{2}{*}{$\begin{array}{l}\% \text { of } \\
\text { relief }\end{array}$} & \multicolumn{6}{|c|}{ Signed rank test } \\
\hline & BT & AT & & & SD & SE & $\mathbf{n}$ & $\mathbf{W}$ & $\mathbf{p}$ & Remarks \\
\hline Kandu (Itching) & 2.43 & 0.05 & 2.38 & 97.90 & 0.49 & 0.07 & 39 & 780 & $<0.001$ & HS \\
\hline Ragata (Redness) & 2.50 & 0.10 & 2.40 & 96.00 & 0.59 & 0.13 & 20 & 210 & $<0.001$ & HS \\
\hline Daha (Burning sensation) & 2.76 & 0.23 & 2.53 & 91.66 & 0.51 & 0.14 & 13 & 91 & $<0.05$ & $\mathrm{~S}$ \\
\hline
\end{tabular}

Table 9: Effect of Gutika Anjana (Group B) on objective criteria of 50 patients.

\begin{tabular}{|c|c|c|c|c|c|c|c|c|c|c|c|}
\hline \multirow{2}{*}{\multicolumn{2}{|c|}{ Objective criteria }} & \multicolumn{2}{|c|}{ Mean } & & \multirow{2}{*}{$\begin{array}{l}\% \text { of } \\
\text { relief }\end{array}$} & \multicolumn{6}{|c|}{ Paired $t$ test } \\
\hline & & BT & AT & & & SD & SE & n & $\mathbf{T}$ & $\mathbf{P}$ & Remarks \\
\hline \multirow{4}{*}{$\begin{array}{c}\text { Palpebral } \\
\text { Conjunctiva }\end{array}$} & Hyperemia & 2.81 & 0.12 & 2.69 & 95.65 & 0.58 & 0.08 & 49 & 32.25 & $<0.001$ & HS \\
\hline & Swelling & 2.33 & 0.00 & 2.33 & 100 & 0.49 & 0.14 & 12 & 16.41 & $<0.001$ & HS \\
\hline & Follicle & 1.00 & 0.25 & 0.75 & 75.00 & 0.50 & 0.25 & 4 & 3.00 & $<0.05$ & $\mathrm{~S}$ \\
\hline & Papillae & 1.00 & 0.16 & 0.84 & 83.67 & 0.37 & 0.05 & 49 & 15.68 & $<0.001$ & HS \\
\hline Bul & Hyperemia & 2.76 & 0.15 & 2.61 & 94.28 & 0.54 & 0.09 & 38 & 27.01 & $<0.001$ & HS \\
\hline Conjunctiva & Chemosis & 0.00 & 0.00 & 0.00 & 0.00 & 0.00 & 0.00 & 0 & - & - & - \\
\hline
\end{tabular}

\section{Comparison between Group A and Group B} $(\mathrm{p}>0.05)$.

Statistically insignificant difference was found in all the signs and symptoms between the two groups

Table 10: Comparative effect of therapy between Punarnavadi eye drop (Group A) Gutika Anjana (Group B) on chief complaints

\begin{tabular}{|c|c|c|c|c|c|c|c|c|c|}
\hline \multirow[b]{2}{*}{ Chief complaints } & \multirow[b]{2}{*}{ Group } & \multirow[b]{2}{*}{$\mathbf{N}$} & \multirow{2}{*}{$\begin{array}{l}\text { Mean } \\
\text { diff. }\end{array}$} & \multicolumn{6}{|c|}{ Mann Whitney Test } \\
\hline & & & & SD & SE & $\mathbf{U}$ & $\mathbf{U}^{\prime}$ & $\mathbf{P}$ & Significance \\
\hline \multirow{2}{*}{ Toda(Pricking pain) } & A & 40 & 2.57 & 0.67 & 0.10 & \multirow{2}{*}{772} & \multirow{2}{*}{1028} & \multirow{2}{*}{$>0.05$} & \multirow{2}{*}{ IS } \\
\hline & $\mathrm{B}$ & 45 & 2.35 & 0.64 & 0.09 & & & & \\
\hline \multirow{2}{*}{ Stambha (Stiffness of lids) } & A & 28 & 2.75 & 0.64 & 0.12 & \multirow{2}{*}{300} & \multirow{2}{*}{540} & \multirow{2}{*}{$>0.05$} & \multirow{2}{*}{ IS } \\
\hline & $\mathrm{B}$ & 30 & 2.40 & 0.49 & 0.09 & & & & \\
\hline \multirow{2}{*}{ Sangharsha (Foreign body sensation) } & A & 46 & 2.65 & 0.56 & 0.08 & \multirow{2}{*}{695} & \multirow{2}{*}{1099} & \multirow{2}{*}{$>0.05$} & \multirow{2}{*}{ IS } \\
\hline & $\mathrm{B}$ & 39 & 2.41 & 0.54 & 0.08 & & & & \\
\hline \multirow{2}{*}{ Parushya(Roughness) } & $\mathrm{A}$ & 20 & 2.45 & 0.51 & 0.11 & \multirow{2}{*}{142} & \multirow{2}{*}{177} & \multirow{2}{*}{$>0.05$} & \multirow{2}{*}{ IS } \\
\hline & $\mathrm{B}$ & 16 & 2.31 & 0.60 & 0.15 & & & & \\
\hline \multirow{2}{*}{ Shiroabhitapa(Headache) } & A & 34 & 2.29 & 0.57 & 0.09 & \multirow{2}{*}{596} & \multirow{2}{*}{662} & \multirow{2}{*}{$>0.05$} & \multirow{2}{*}{ IS } \\
\hline & $\mathrm{B}$ & 37 & 2.35 & 0.58 & 0.09 & & & & \\
\hline \multirow{2}{*}{ Vishushka Bhava(Dryness - feeling) } & A & 35 & 2.85 & 0.64 & 0.10 & \multirow{2}{*}{550} & \multirow{2}{*}{675} & \multirow{2}{*}{$>0.05$} & \multirow{2}{*}{ IS } \\
\hline & $\mathrm{B}$ & 35 & 2.71 & 0.71 & 0.12 & & & & \\
\hline Shishirasruta(Cold lacrimation) & A & 15 & 2.66 & 0.72 & 0.18 & 62 & 73 & $>005$ & IS \\
\hline & $\mathrm{B}$ & 9 & 2.55 & 1.01 & 0.33 & 02 & 13 & & 15 \\
\hline Achchasruta (clean/ watery discharge) & A & 51 & 2.94 & 0.70 & 0.09 & 1020 & 1428 & $>0.05$ & IS \\
\hline & $\mathrm{B}$ & 48 & 2.70 & 0.71 & 0.10 & $10 \angle 0$ & 1420 & -0.05 & \\
\hline
\end{tabular}




\begin{tabular}{|c|c|c|c|c|c|c|c|c|c|}
\hline \multirow{2}{*}{ Alpa Shopha (Mild chemosis) } & A & 27 & 2.37 & 0.56 & 0.10 & \multirow{2}{*}{258} & \multirow{2}{*}{308} & \multirow{2}{*}{$>0.05$} & \multirow{2}{*}{ IS } \\
\hline & B & 21 & 2.47 & 0.51 & 0.11 & & & & \\
\hline \multirow{2}{*}{$\begin{array}{c}\text { Jantunam Iva Sarpanam (Crawling } \\
\text { sensation) }\end{array}$} & A & 46 & 2.60 & 0.53 & 0.07 & \multirow[b]{2}{*}{721} & \multirow[b]{2}{*}{1072} & \multirow{2}{*}{$>0.05$} & \multirow[b]{2}{*}{ IS } \\
\hline & B & 39 & 2.41 & 0.54 & 0.08 & & & & \\
\hline \multirow{2}{*}{$\begin{array}{l}\text { Nimeshonmeshana Krichchata } \\
\text { (Difficulty in lid movement) }\end{array}$} & A & 7 & 2.42 & 0.53 & 0.20 & \multirow[b]{2}{*}{21} & \multirow[b]{2}{*}{28} & \multirow{2}{*}{$>0.05$} & \multirow[b]{2}{*}{ IS } \\
\hline & B & 7 & 2.28 & 0.48 & 0.18 & & & & \\
\hline \multirow{2}{*}{$\begin{array}{c}\text { Akshyadhmantam Iva Bhati (Feeling of } \\
\text { distended eyes) }\end{array}$} & A & 37 & 2.24 & 0.64 & 0.10 & \multirow[b]{2}{*}{599} & \multirow[b]{2}{*}{732} & \multirow[b]{2}{*}{$>0.05$} & \multirow{2}{*}{ IS } \\
\hline & B & 36 & 2.36 & 0.63 & 0.10 & & & & \\
\hline \multirow{2}{*}{ Shushka Dushika (Dry discharge) } & A & 46 & 2.89 & 0.64 & 0.09 & \multirow{2}{*}{844} & \multirow{2}{*}{1088} & \multirow{2}{*}{$>0.05$} & \multirow{2}{*}{ IS } \\
\hline & B & 42 & 2.73 & 0.66 & 0.10 & & & & \\
\hline \multirow{2}{*}{ Alpa Dushika (Scanty discharge) } & A & 44 & 2.77 & 0.60 & 0.09 & \multirow{2}{*}{876} & \multirow{2}{*}{1016} & \multirow{2}{*}{$>0.05$} & \multirow{2}{*}{ IS } \\
\hline & B & 43 & 2.69 & 0.67 & 0.10 & & & & \\
\hline \multirow{2}{*}{ Chala Ruja (Reffered pain) } & A & 20 & 2.40 & 0.59 & 0.13 & \multirow{2}{*}{251} & \multirow{2}{*}{264} & \multirow{2}{*}{$>0.05$} & \multirow{2}{*}{ IS } \\
\hline & $\mathrm{B}$ & 26 & 2.38 & 0.49 & 0.09 & & & & \\
\hline
\end{tabular}

U- Mann Whitney test

Table 11: Comparative effect of therapy between Punarnavadi eye drop (Group A) Gutika Anjana (Group B) on associated symptoms

\begin{tabular}{|c|c|c|c|c|c|c|c|c|c|}
\hline \multirow{2}{*}{$\begin{array}{l}\text { Associated } \\
\text { symptoms }\end{array}$} & \multirow{2}{*}{ Group } & \multirow{2}{*}{$\mathbf{N}$} & \multirow{2}{*}{$\begin{array}{c}\text { Mean } \\
\text { diff. }\end{array}$} & \multicolumn{6}{|c|}{ Mann Whitney Test } \\
\hline & & & & SD & SE & $\mathbf{U}$ & $\mathbf{U}^{\prime}$ & $\mathbf{P}$ & Remarks \\
\hline \multirow{2}{*}{$\begin{array}{c}\text { Kandu } \\
\text { (Itching) }\end{array}$} & $\mathrm{A}$ & 45 & 2.62 & 0.57 & 0.08 & \multirow{2}{*}{673} & \multirow{2}{*}{1081} & \multirow{2}{*}{$>0.05$} & \multirow{2}{*}{ IS } \\
\hline & $\mathrm{B}$ & 39 & 2.38 & 0.49 & 0.07 & & & & \\
\hline \multirow{2}{*}{$\begin{array}{c}\text { Ragata } \\
\text { (Redness) }\end{array}$} & A & 18 & 2.38 & 0.50 & 0.11 & \multirow{2}{*}{174} & \multirow{2}{*}{185} & \multirow{2}{*}{$>0.05$} & \multirow{2}{*}{ IS } \\
\hline & $\mathrm{B}$ & 20 & 2.40 & 0.59 & 0.13 & & & & \\
\hline \multirow{2}{*}{$\begin{array}{c}\text { Daha } \\
\text { (Burning sensation) }\end{array}$} & A & 9 & 2.66 & 0.50 & 0.16 & \multirow{2}{*}{51} & \multirow{2}{*}{66} & \multirow{2}{*}{$>0.05$} & \multirow{2}{*}{ IS } \\
\hline & $\mathrm{B}$ & 13 & 2.53 & 0.51 & 0.14 & & & & \\
\hline
\end{tabular}

Table 12: Comparative effect of therapy between Punarnavadi eye drop (Group A) Gutika Anjana (Group B) on objective criteria

\begin{tabular}{|c|c|c|c|c|c|c|c|c|c|}
\hline \multirow{2}{*}{\multicolumn{2}{|c|}{ Objective criteria }} & \multirow{2}{*}{ Group } & \multirow{2}{*}{$\mathbf{N}$} & \multirow{2}{*}{$\begin{array}{l}\text { Mean } \\
\text { diff. }\end{array}$} & \multicolumn{5}{|c|}{ Unpaired t-Test } \\
\hline & & & & & SD & SE & $\mathbf{T}$ & $\mathbf{P}$ & Remarks \\
\hline \multirow{8}{*}{$\begin{array}{l}\text { Palpebral } \\
\text { Conjunctiva }\end{array}$} & \multirow{2}{*}{ Hyperemia } & A & 50 & 2.68 & 0.51 & 0.07 & \multirow{2}{*}{0.126} & \multirow{2}{*}{$>0.05$} & \multirow{2}{*}{$1 \mathrm{~S}$} \\
\hline & & B & 49 & 2.69 & 0.58 & 0.08 & & & \\
\hline & \multirow{2}{*}{ Swelling } & A & 20 & 2.15 & 0.58 & 0.13 & \multirow{2}{*}{0.906} & \multirow{2}{*}{$>0.05$} & \multirow{2}{*}{$1 \mathrm{~S}$} \\
\hline & & B & 12 & 2.33 & 0.49 & 0.14 & & & \\
\hline & \multirow{2}{*}{ Follicle } & A & 9 & 0.55 & 0.52 & 0.17 & \multirow{2}{*}{0.622} & \multirow{2}{*}{$>0.05$} & \multirow{2}{*}{$1 \mathrm{~S}$} \\
\hline & & B & 4 & 0.75 & 0.50 & 0.25 & & & \\
\hline & \multirow{2}{*}{ Papillae } & A & 46 & 0.76 & 0.48 & 0.07 & \multirow{2}{*}{0.863} & \multirow{2}{*}{$>0.05$} & \multirow{2}{*}{$1 \mathrm{~S}$} \\
\hline & & $\mathrm{B}$ & 49 & 0.83 & 0.37 & 0.05 & & & \\
\hline \multirow{2}{*}{$\begin{array}{c}\text { Bulbar } \\
\text { Conjunctiva }\end{array}$} & \multirow{2}{*}{ Hyperemia } & A & 38 & 2.68 & 0.57 & 0.09 & \multirow{2}{*}{0.58} & \multirow{2}{*}{$>0.05$} & \multirow{2}{*}{$1 \mathrm{~S}$} \\
\hline & & $\mathrm{B}$ & 38 & 2.60 & 0.59 & 0.09 & & & \\
\hline
\end{tabular}

\section{Overall effect of therapy}

In Group A, out of 51 patients, 96.08\% got complete relief after the completion of treatment, $03.92 \%$ got marked improvement. In Group B, out of 50 patients, $98 \%$ got complete relief after the completion of treatment, $2 \%$ got marked improvement.

From overall effect of therapy it can be concluded that Group B has cured more percentage of patients than Group A. It helps in breaking the pathogenesis of the disease and stop the progression of disease.

\section{Graph 1: Showing percentage of overall effect of therapy}

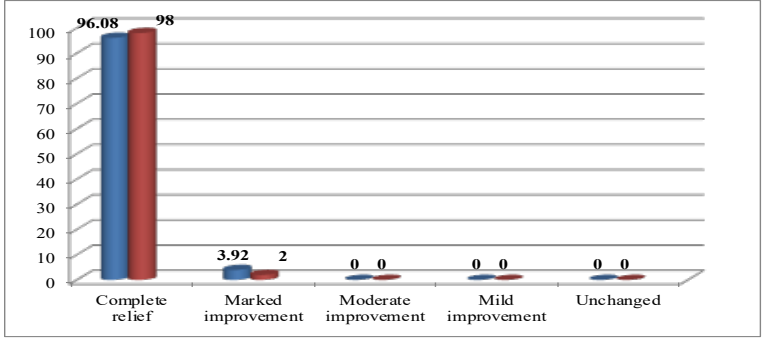

\section{Discussion}

Allergic conjunctivitis is one of the most common problems seen by ophthalmologists worldwide. Allergic conjunctivitis is estimated to affect 20 percent of the population on an annual basis and approximately one-half of these people have a personal or family history of atopy(4). In Jamnagar, due to the natural dry and dusty weather, heavy industrialization, individuals of this area are prone to allergy of eye. Patient suffering from allergic conjunctivitis is attending the OPD of Shalakya Tantra department of I.P.G.T \& R.A for the treatment of allergic conjunctivitis. Therefore, a timely diagnosis and management is required to seize the progression of this disease.

Modern management include topical antihistamines, mast cell stabilizers, nonsteroidal antiinflammatory drugs (NSAIDs), and corticosteroids. But they have their own limitations. By considering all these limitations of modern science, the discussion pointed towards Ayurveda. Is there any formulation available in 
traditional system of Ayurveda which can control Inflammation, promote immune-modulation of ocular surface, free from preservatives and cost effective? This thought lead to this clinical study to find out an Ayurvedic formulation which can manage the Allergic conjunctivitis (Vataja Abhishyanda) efficiently by curing the underlying pathology and improving the ocular surface defence mechanism.

According to Ayurveda the instilled medicine (Aschyotana) will penetrate into Akshikosha Srotas, Shira Srotas, Ghrana Srotas and Mukha Srotas of the Urdhvanga Bhaga and remove the Mala present there. After absorption of Eye Drop may undergo systemic distribution primarily by nasal mucosa absorption and possible by local ocular distribution by transcorneal/ transconjunctival absorption and metabolism of eye drops by various enzymes. Hence after instillation of eye drops, these fluids undergo absorption, distribution and metabolism. So effect of eye drops is local as well as systemic.

Anjana have micro particles which may be deposited in the cul-de-sac and thereby increase the bioavailability to enhance ocular absorption. The ocular absorption of Anjana may initiate though the conjunctiva and cornea. Mainly lipophilic active ingredients may absorb through the cornea by transcellular pathway and hyrophilics from the conjunctiva by paracellular pathway. Once it crosses the conjunctiva (mainly hydrophilics); the sclera is more permeable and it allows drugs to penetrate the other interior structures of the eye i.e. ciliary body, iris, aqueous humour, lens, vitreous etc.

Both the drugs i.e. Punarnavadi eye drops (Punarnava(5),(6), Saindhava(7), Sita(8) and Sphatika(9) and Gutika Anjana (Shunthi(10), Pippali(11), Gairika(12) and Saindhava) have Tridoshashamana effect. They are Madhura, Katu, Kashaya in Rasa, Snigdha Guna, Ushna Virya and Madhura Vipaka. All these factors help to break the Samprapti of Vataja Abhishyanda and relieve the symptoms like Toda, Stambha, Sangharsa, Kandu, Daha, Ragata, etc. They have Rasayana, Chakshushya, Shothhara, Raktastambhaka, Deepana, Pachana, Vatanulomana property. It helps to relieve the inflammatory changes that decrease the signs like hyperemia, swelling, papillae, follicles present in conjunctiva.

\section{Conclusion}

The clinical features of Vataja Abhishyanda explained in all classical texts resembles with most of sign and symptoms of allergic conjunctivitis. Punarnavadi eye drop and Gutika Anjana both have showed excellent effect on signs and symptoms of allergic conjunctivitis on the basis of percentage relief.
But Gutika Anjana showed better result in maximum sign and symptoms on the basis of percentage. The reason may be Anjana has maximum absorption due to more contact of time with the tissue which is responsible for better bioavailability. No adverse reactions were reported in any of the patients during the entire study period and follow up.

Financial support and sponsorship

I.P.G.T. and R.A Jamnagar, Gujarat.

\section{Conflicts of interest}

There are no conflicts of interest.

\section{Reference}

1. http:// www.hqlo.com/content/3/1/67 dated 10-09-2019 time 12:05 IST

2. Bogacka E. [Epidemiology of allergic eye diseases]. Pol Merkur Lekarski.2003;14(84);714-15

3. Indradev tripathi, Gadanigraha 3rd Vol, Chaukhambha Sanskrit Sansthan, Varanasi 2016, 95p.

4. Conjunctivitis: Differentiating Allergic, Bacterial \& Viral Conjunctivitis". conjunctivitis.blogspot.com. dated 03-08-2019 time 13:10 IST)

5. Sri Brahmasankara Misra et al. Bhavaprakasha 7ed Vol 1, Chaukhambha Sanskrit Sansthan, Varanasi 2015, 423p.

6. The Ayurvedic Pharmacopoiea of India, Ministry of Health and family welfare, Department of AYUSH, Government of India, New Delhi. Part I, Vol I, $126-128 \mathrm{p}$.

7. Sri Chakrapanidatta Ayurveda Deepika Vyakhya on Charaka Samhita, by Krishnadas Academy, Varanasi, 1998, 284p.

8. Sri Brahmasankara Misra et al. Bhavaprakasha 7ed Vol 1, Chaukhambha Sanskrit Sansthan, Varanasi 2006, 794p.

9. Professor Dattatreya Ananta Kulkarni, Vijnanabodhini tika on Rasa Ratna Samuchaya, Meherchand Lachmandas Publications, New Delhi,1998.

10. Sameer Mlhotra et al, Medicinal properties of Ginger( Zingiber officinale Rosc.), National product radiance Vol 2(6), Nov-Dec 2003.

11. Apurva Priyadarshi, Pharmacognostical and phytochemical analysis of Pippali (Piper longum Linn.), The Pharma Innovation Journal 2018; 7(6): 286-289 ISSN (E): 2277- 7695 ISSN (P): 2349-8242.

12. Lekshmipriya S. et al, A Critical Review On Gairikam, World Journal Of Pharmacy And Pharmaceutical Sciences, Volume 6, Issue 9, 1791-1799. 